\title{
Crise Económica em Portugal: Evolução da Incidência de Depressão e Correlação com o Desemprego
}

\section{Economic Crisis in Portugal: Trajectory of the Incidence of Depression and Correlation With Unemployment}

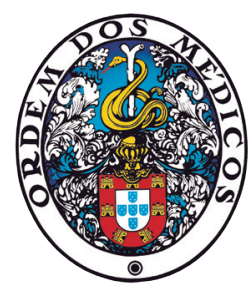

\author{
Inês Laplanche COELHO ${ }^{1}$, Mafalda SOUSA-UVA ${ }^{2,3}$, Nuno PINA ${ }^{4}$, Sara MARQUES ${ }^{4}$, Carlos MATIAS-DIAS ${ }^{2,3}$, \\ Ana Paula RODRIGUES $\square^{2}$
}

Acta Med Port 2021 Apr;34(4):278-282 - https://doi.org/10.20344/amp.13574

\section{RESUMO}

Introdução: Estudos anteriores verificaram um aumento da taxa de incidência de depressão entre 2007 e 2013 em Portugal, a qual se correlacionou positivamente com a taxa de desemprego, nomeadamente, em homens. Tal facto levantou a hipótese desse aumento se encontrar relacionado com a situação de crise económica à data. No sentido de testar esta hipótese, este estudo teve como objetivo investigar se a correlação entre taxa de desemprego e incidência de depressão se manteve no período de recuperação económica pós-crise em Portugal (2016 - 2018).

Material e Métodos: Realizou-se um estudo ecológico, utilizando dados da rede Médicos Sentinela relativos à incidência de depressão (primeiros episódios e recidiva) e dados do Instituto Nacional de Estatística sobre a taxa de desemprego na população portuguesa. O coeficiente de correlação foi estimado através de regressão linear e os resultados foram desagregados por sexo.

Resultados: Entre 2016 e 2018, verificou-se um decréscimo consistente da incidência de depressão em ambos os sexos. Durante o período 1995 - 2018, observou-se uma correlação positiva entre desemprego e depressão, sendo o seu coeficiente de 0,833 ( $p=$ $0,005)$ nos homens e de $0,742(p=0,022)$ nas mulheres.

Discussão: A redução da taxa de incidência de depressão em ambos os sexos, observada entre 2016 e 2018, corrobora a existência da correlação positiva entre desemprego e depressão na população portuguesa, observada anteriormente em 2007 - 2013.

Conclusão: Este estudo reforça a necessidade de monitorização da ocorrência de doença mental na população portuguesa, em especial em momentos de maior vulnerabilidade social, para instituição de medidas preventivas como forma de mitigar o impacto de futuras crises económicas.

Palavras-chave: Cuidados de Saúde Primários; Depressão Vigilância de Evento Sentinela; Desemprego; Portugal; Recessão Económica

\section{ABSTRACT}

Introduction: Previous studies have found an increase in the incidence rate of depression between 2007 - 2013 in Portugal, with a positive correlation with the unemployment rate, namely, in men. So, it was hypothesized that this increase is related with the situation of economic crisis. This study aimed to investigate if the correlation between unemployment rates and the incidence of depression is maintained in the post-crisis period of economic recovery in Portugal $(2016-2018)$.

Material and Methods: An ecological study was carried out, using data from the General Practitioners Sentinel Network concerning depression incidence (first episodes and relapses) and data from the National Statistics Institute on unemployment rates in the Portuguese population. The correlation coefficient was estimated using linear regression and the results were disaggregated by sex.

Results: Between 2016 and 2018, there was a consistent decrease in the incidence of depression in both sexes. During the $1995-$ 2018 period, a positive correlation was observed between unemployment and depression, with a coefficient of $0.833(p=0.005)$ in males and of $0.742(p=0.022)$ in females.

Discussion: The reduction in the incidence of depression in both sexes observed between 2016 - 2018 corroborates a positive correlation between unemployment and depression in the Portuguese population, previously observed between 2007 - 2013.

Conclusion: This study highlights the need to monitor the occurrence of mental illness in the Portuguese population, especially in moments of greatest social vulnerability in order to establish preventive measures, as a way to mitigate the impact of future economic crises.

Keywords: Depression; Economic Recession; Portugal; Primary Health Care; Sentinel Surveillance; Unemployment

\section{INTRODUÇÃO}

Sabe-se hoje que a crise económica que atingiu a Europa em 2007 teve um impacto negativo na saúde das populações, quer pelo impacto económico direto no orçamento das famílias, quer indiretamente por aplicação de medidas de austeridade sobre o sistema de saúde..$^{1,2}$

Portugal encontra-se entre os países mais afetados por esta crise económica, tendo sido necessário apoio financeiro internacional adicional, de 2011 a 2014, sob a supervisão do Fundo Monetário Internacional, Banco Central Europeu e da Comissão Europeia. De 2011 a 2013, o país perdeu aproximadamente $7 \%$ do produto interno bruto (PIB) e a taxa anual de desemprego aumentou de $8,8 \%$ em 2008 para $16,4 \%$ em 2013, sendo uma das mais elevadas da Europa. ${ }^{3} \mathrm{O}$ setor da saúde também foi atingido com o

1. Unidade de Saúde Familiar do Dafundo. Agrupamento de Centros de Saúde Lisboa Ocidental e Oeiras. Oeiras. Portugal.

2. Departamento de Epidemiologia. Instituto Nacional de Saúde Doutor Ricardo Jorge. Lisboa. Portugal.

3. Centro de Investigação em Saúde Pública. Escola Nacional de Saúde Pública. Universidade NOVA de Lisboa. Lisboa. Portugal.

4. Unidade de Saúde Familiar Tondela. Agrupamento de Centros de Saúde Dão-Lafões. Tondela. Portugal.

$\triangle$ Autor correspondente: Ana Paula Rodrigues. ana.rodrigues@insa.min-saude.pt

Recebido: 10 de fevereiro de 2020 - Aceite: 06 de julho de 2020 - First published: 28 de outubro de 2020 - Online issue published: 01 de abril de 2021 Copyright $\odot$ Ordem dos Médicos 2021 
aumento das barreiras no acesso aos cuidados de saúde, bem como com desinvestimentos em equipamentos e infraestruturas de saúde. ${ }^{4}$

A depressão major é mais frequente em doentes com fatores de risco específicos. Estão descritas três vias principais que interagem e podem explicar o desenvolvimento da doença: os fatores intrínsecos como o neuroticismo ou a baixa auto-estima, os fatores extrínsecos como o consumo abusivo de substâncias ou outros eventos adversos de vida, como por exemplo, os problemas conjugais ou o baixo apoio social. ${ }^{5} \mathrm{~A}$ relação entre fatores socioeconómicos e o estado de saúde mental também tem sido amplamente estudada, sendo que é conhecida a relação entre a instabilidade socioeconómica e o desenvolvimento de problemas de saúde mental. ${ }^{6,7}$ Períodos de recessão económica contribuem para uma maior frequência de problemas de saúde mental, tais como depressão, perturbações de ansiedade, abuso de substâncias e comportamento suicida por aumento do desemprego, dificuldades financeiras, endividamento e problemas relacionados ao trabalho. ${ }^{8}$ Em particular, o desemprego crescente durante períodos de crise económica tem sido utilizado como um dos indicadores macroeconómicos associado à deterioração da saúde mental. ${ }^{8}$

Estudos anteriores realizados em Portugal pela nossa equipa de investigação observaram uma correlação positiva entre a taxa de desemprego e a taxa de incidência de depressão no sexo masculino durante o período de crise económica (entre 2007 e 2013). Colocou-se, então, a hipótese de que o aumento observado da taxa de incidência de depressão em Portugal se pudesse encontrar relacionado com a situação de crise económica. ${ }^{9}$

Até ao início da pandemia de COVID-19, Portugal estava a recuperar lentamente da crise, com um aumento progressivo da taxa de crescimento real do PIB, desde 2014 com valores positivos, e uma diminuição da taxa de desemprego. ${ }^{10}$

A condução de estudos sobre o impacto de crises económicas na saúde mental é cíclica, tendo-se verificado uma tendência de aumento durante o período de recessão e imediatamente após. ${ }^{11}$ Porém, verifica-se uma escassez de estudos que investiguem o impacto do período de recuperação económica (pós-crise) na saúde mental. A relevância destes estudos assenta na necessidade de conhecimento sobre o eventual agravamento ou melhoria da situação de saúde mental das populações durante esse período, bem como da análise da evolução da frequência dos problemas de saúde mental ao longo dos períodos que antecedem e sucedem uma crise socioeconómica, nomeadamente, para investigar se no período pós crise retomam os valores que apresentaram no período pré-crise.

Por forma a responder a esta lacuna de conhecimento em Portugal, o presente estudo teve como objetivo descrever a evolução da taxa de incidência de depressão nos períodos antes, durante e após a crise económica e verificar se a correlação entre a taxa de desemprego e a incidência de depressão, encontrada anteriormente durante o período de crise económica, se mantém incluindo o período pós- -crise (2016 a 2018).

\section{MATERIAL E MÉTODOS}

Desenvolveu-se um estudo ecológico no qual se descreveu a evolução da taxa de incidência de depressão nos anos 1995, 1996, 1997, 2004, 2012, 2013, 2016, 2017 e 2018 e se correlacionou com a taxa de desemprego em Portugal.

Os dados de incidência de depressão (primeiros episódios e recidiva) foram obtidos da rede Médicos-Sentinela e foram utilizados os dados relativos à taxa de desemprego disponíveis nas estatísticas oficiais do Instituto Nacional de Estatística (INE). ${ }^{10}$

A rede Médicos-Sentinela é um sistema de observação em saúde constituído por médicos de família do Serviço Nacional de Saúde que notificam voluntariamente, de modo contínuo e sistemático, um conjunto pré-determinado de eventos de saúde ocorridos nos seus utentes.

O numerador utilizado para cálculo da taxa de incidência de depressão foi o número de novos diagnósticos de depressão (primeiros casos na vida do utente e recidiva) e o denominador a população sob observação efetiva na rede Médicos-Sentinela. A população sob observação efetiva resulta do somatório das listas de utentes dos médicos ativos em cada semana, isto é, dos médicos que notificaram casos em cada semana. Nos anos em estudo, a população sob observação na Rede variou entre um mínimo de 28184 indivíduos em 2013 e um máximo de 164676 indivíduos em 1995.

Os eventos em notificação pela rede Médicos Sentinela variam em cada ano; assim, a depressão foi considerada como evento a notificar nos anos de 1995, 1996, 1997, 2004, 2012, 2013, 2016, 2017 e 2018, motivo pelo qual apenas se encontram disponíveis as taxas de incidência para esses anos. Em todos os anos em estudo, a definição de caso de depressão usada foi baseada no melhor conhecimento clínico, isto é, seguindo os mesmos critérios usados na abordagem diagnóstica e terapêutica do doente, existindo ainda um manual de procedimentos para uniformizar o modo como era feita a notificação dos casos.

Nos anos 1995 - 1997 e 2017, 2018 estiveram em notificação os casos de depressão (primeiro episódio na vida do utente e episódios seguintes), enquanto que em 2004, 2012 e 2013 o evento em notificação foi a consulta relacionada com depressão. Assim, nestes anos, foram consideradas apenas as primeiras consultas de depressão correspondentes a primeiros episódios ou episódios seguintes.

A taxa de incidência de depressão foi padronizada pelo método direto usando como população padrão, a população padrão europeia de 1976.

A correlação entre a taxa de desemprego e a taxa de incidência de depressão foi estimada pela aplicação de um modelo de regressão linear, com desagregação por sexo. Foi verificada a normalidade dos dados através da exploração visual do histograma e dos gráficos de resíduos. Considerou-se um nível de significância estatística de $5 \%$. A análise foi conduzida no software de análise estatística 
SPSS, versão 22.

A recolha de dados anonimizados no âmbito das atividades de vigilância da rede Médicos Sentinela obteve parecer favorável da Comissão de Ética do Instituto Nacional de Saúde e da Comissão Nacional de Protecção de Dados. Estes dados são recolhidos primariamente com o objetivo de monitorizar vários problemas de saúde, pelo que neste trabalho foi feita uma utilização de dados secundários agregados e anonimizados.

\section{RESULTADOS}

Verificou-se um aumento acentuado da taxa de incidência de depressão em ambos os sexos, a partir do ano de 2004. Do total de anos observados, o de 2013 foi aquele em que a taxa de incidência foi mais elevada, observando-se um decréscimo consistente, em ambos os sexos, a partir desse ano. O sexo feminino apresentou, em todos os anos, taxas de incidência mais elevadas (Tabela 1).

Tabela 1 - Incidência de depressão (primeiros episódios e recidiva) em Portugal, em homens e mulheres (/100 000)

\begin{tabular}{|c|c|c|c|c|}
\hline \multirow[b]{2}{*}{ Ano } & \multicolumn{2}{|c|}{ Masculino } & \multicolumn{2}{|c|}{ Feminino } \\
\hline & $\begin{array}{r}\text { Bruta } \\
(/ 100000)\end{array}$ & $\begin{array}{r}\text { Padronizada } \\
(/ 100000)\end{array}$ & $\begin{array}{r}\text { Bruta } \\
(/ 100000)\end{array}$ & $\begin{array}{r}\text { Padronizada } \\
(/ 100 \text { 000) }\end{array}$ \\
\hline 1995 & 178,0 & 181,1 & 789,0 & 788,2 \\
\hline 1996 & 143,3 & 143,7 & 879,9 & 882,5 \\
\hline 1997 & 172,3 & 164,7 & 841,3 & 828,2 \\
\hline 2004 & 476,6 & 451,8 & 1963,9 & 1873,3 \\
\hline 2012 & 571,5 & 539,5 & 2136,0 & 1968,0 \\
\hline 2013 & 731,4 & 668,3 & 2103,5 & 1928,7 \\
\hline 2016 & 518,1 & 472,2 & 1632,0 & 1469,1 \\
\hline 2017 & 471,6 & 411,9 & 1467,8 & 1328,5 \\
\hline 2018 & 269,1 & 247,6 & 820,7 & 740,4 \\
\hline
\end{tabular}

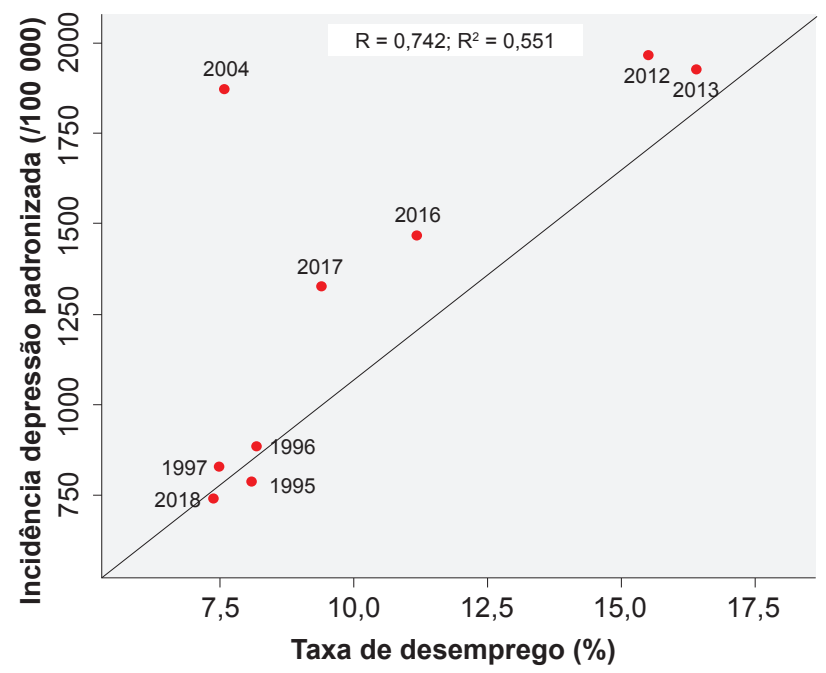

Figura 1 - Gráfico de dispersão entre desemprego e incidência de depressão no sexo masculino em Portugal nos anos de 1995, 1996, 1997, 2004, 2012, 2013, 2016, 2017 e 2018 (únicos anos com notificação de depressão na rede Médicos Sentinela)
Observou-se uma maior correlação entre desemprego e depressão no sexo masculino $(R=0,833, p=0,005)$ relativamente ao sexo feminino $(R=0,742, p=0,022)$, sendo ambas as correlações estatisticamente significativas (Tabela 2, Fig.s 1 e 2).

Entre 1995 e 2018, no sexo masculino, observou-se um aumento de 37,8 casos de depressão por 100000 habitantes por cada aumento unitário da taxa de desemprego, sendo que no sexo feminino este aumento foi de 110,2 casos por 100000 habitantes.

\section{DISCUSSÃO}

Os resultados do presente estudo corroboram a existência de uma correlação positiva entre desemprego e depressão na população portuguesa, observada pelo nosso grupo de investigação até $2013 .{ }^{9}$ Este trabalho de monitorização pretendia verificar se a correlação observada se mantinha após a crise, ou seja, no período de recuperação económica. Com a integração de dados referentes a anos posteriores (2016, 2017 e 2018), anos de recuperação económica com menores taxas de desemprego, este estudo demonstrou uma redução da taxa de incidência de depressão, em ambos os sexos.

A diferença encontrada na incidência de depressão entre sexos encontra-se em concordância com Van de Velde et al que estudou a prevalência de sintomas depressivos em vários países europeus e que encontrou a maior diferença entre mulheres e homens em Portugal. ${ }^{12}$

Tabela 2 - Variação da incidência de depressão (/100 000) por variação unitária da taxa de desemprego em Portugal entre 1995 e 2018

\begin{tabular}{lccc}
\hline Sexo & Variação & IC 95\% & $\boldsymbol{p}$ \\
\hline Masculino & 37,8 & $15,3-60,3$ & 0,005 \\
Feminino & 110,2 & $21,3-199,1$ & 0,022 \\
\hline
\end{tabular}

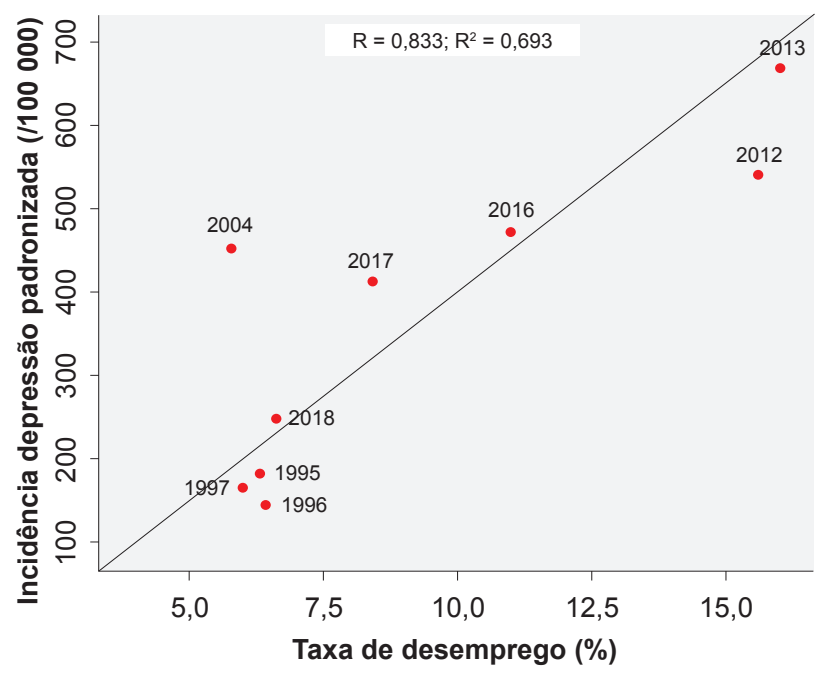

Figura 2 - Gráfico de dispersão entre desemprego e incidência de depressão no sexo feminino em Portugal nos anos de 1995, 1996, 1997, 2004, 2012, 2013, 2016, 2017 e 2018 (únicos anos com notificação de depressão na rede Médicos Sentinela) 
Manteve-se a maior correlação no sexo masculino, tal como observado anteriormente. ${ }^{9} \mathrm{O}$ facto de no estudo anterior a correlação entre desemprego e depressão no sexo feminino não ter sido estatisticamente significativa, pode ter-se devido ao menor número de anos em estudo. ${ }^{9}$ A literatura existente sobre diferenças no impacto do desemprego na saúde mental de homens e mulheres diverge, demonstrando que outros aspetos, nomeadamente sociopolíticos, poderão explicar estas diferenças. ${ }^{13}$ Van de Velde et al formularam a hipótese de que em países do Sul da Europa, como Portugal, a família tenha um peso importante e, como tal, se verifique uma ideologia de género mais tradicional, levando a que os sintomas depressivos das mulheres estejam mais associados a fatores familiares e os sintomas depressivos dos homens mais associados a fatores socioeconómicos, como o desemprego, apesar de tal não se ter verificado. ${ }^{12}$ Nesse estudo, foi encontrada uma associação entre níveis de depressão e fatores socioeconómicos, tanto em mulheres como em homens, mas não com fatores familiares. ${ }^{12}$ Outro estudo demonstrou menores níveis de bem-estar mental nos desempregados em países do Sul da Europa, caraterizados por um sistema de proteção social com lacunas no apoio aos desempregados. ${ }^{13}$ Adicionalmente, os mesmos autores descrevem que elevadas taxas de desemprego vigentes durante uma crise económica limitam o poder negocial dos trabalhadores e dos sindicatos, aumentando o trabalho precário e aumentando a insegurança de quem está empregado, com consequente aceitação de piores condições de trabalho, as quais também poderão afetar o bem-estar mental da população empregada. ${ }^{13}$ As taxas de desemprego parecem funcionar como um marcador do ambiente e bem-estar económico da população. ${ }^{11,14}$

Como limitações deste estudo importa referir que apenas permite estimar a proporção de utentes de cuidados de saúde primários que recorreram à consulta por depressão, em vez de uma estimativa da real incidência de depressão na população portuguesa. Pode existir ainda um viés de seleção já que os médicos de família que integram a rede constituem uma amostra de conveniência, não tendo sido selecionados aleatoriamente. Estes resultados devem ser interpretados tendo em conta a possibilidade de variação da notificação de casos de depressão na população da rede Médicos Sentinela, da definição e o reduzido número de anos de estudo. De notar que o desenho de estudo ecológico não permite estabelecer relações causais e a nível do indivíduo entre a variável de exposição (desemprego) e a variável resultado (depressão) e que a ausência de controlo para o confundimento por variáveis tais como o rendimento médio das famílias, a acessibilidade aos cui- dados de saúde, área de residência e idade pode comportar um erro com implicações na validade deste estudo. No sexo masculino, a percentagem de variância explicada é de aproximadamente $69 \%\left(R^{2}\right)$ e no sexo feminino é de $55 \%$ $\left(R^{2}\right)$, o que, de facto, indica que outros fatores podem explicar a percentagem remanescente.

Apesar das limitações referidas e de acordo com o nosso conhecimento, este é o primeiro trabalho cujos resultados demonstram o impacto positivo da recuperação económica em Portugal (através da diminuição das taxas de desemprego) na saúde mental dos portugueses. Drydakis comparou períodos pré e intra-crise na Grécia e concluiu que a perda de emprego é mais prejudicial para a saúde mental nos períodos marcados por altas taxas de desemprego. ${ }^{15}$

\section{CONCLUSÃO}

O presente estudo verificou uma redução da taxa de incidência de depressão no período pós crise, em ambos os sexos, bem como uma correlação positiva da mesma com a taxa de desemprego na população portuguesa. Apesar da melhoria dos índices de crescimento económico em Portugal, os episódios de recessão tendem a ser cíclicos e impõe-se a necessidade de refletirmos sobre medidas preventivas que possam minimizar o impacto de uma futura crise económica na saúde mental dos portugueses. Estes resultados indicam, ainda, a necessidade de manter sob monitorização a ocorrência de doença mental na população portuguesa, em especial em momentos de maior vulnerabilidade social.

\section{PROTEÇÃO DE PESSOAS E ANIMAIS}

Os autores declaram que os procedimentos seguidos estavam de acordo com os regulamentos estabelecidos pelos responsáveis da Comissão de Investigação Clínica e Ética e de acordo com a Declaração de Helsínquia da Associação Médica Mundial atualizada em 2013.

\section{CONFIDENCIALIDADE DOS DADOS}

Os autores declaram ter seguido os protocolos do seu centro de trabalho acerca da publicação de dados.

\section{CONFLITOS DE INTERESSE}

Os autores declaram não ter conflitos de interesses relacionados com o presente trabalho.

\section{FONTES DE FINANCIAMENTO}

Este trabalho não recebeu qualquer tipo de suporte financeiro de nenhuma entidade no domínio público ou privado.

\section{REFERÊNCIAS}

1. Modrek S, Stuckler D, McKee M, Cullen MR, Basu S. A review of health consequences of recessions internationally and a synthesis of the US response during the great recession. Public Health Rev. 2013;35:1-33.

2. Thomson S, Figueras J, Evetovits T, Jowett M, Mladovsky P, Maresso $A$, et al. Economic crisis, health systems, and health in Europe: impact

and implications for policy. Copenhagen: World Health Organization Regional Office for Europe; 2014.

3. Antunes A, Frasquilho D, Cardoso G, Pereira N, Silva M, Caldas-deAlmeida JM, et al. Perceived effects of the economic recession on population mental health, well-being and provision of care by primary 
care users and professionals: a qualitative study protocol in Portugal. BMJ Open. 2017;7:e017032.

4. Morais N, Cunha F, Campos F. Financial crisis in Portugal: effects in the health care sector. Int J Health Serv. 2019;49:237-59.

5. Kendler K, Gardner C, Prescott C. Toward a comprehensive developmental model for major depression in men. Am J Psychiatry. 2006;163:115.

6. Herrman H, Saxena S, Moodie R, editors. Promoting mental health: concepts, emerging evidence and practice. Geneva: World Health Organization; 2005.

7. World Health Organization, Regional Office for Europe. Impact of economic crisis on mental health. Copenhagen: WHO; 2011.

8. Frasquilho D, Matos MG, Salonna F, Guerreiro D, Storti CC, Gaspar T, et al. Mental health outcomes in times of economic recession: a systematic literature review. BMC Public Health. 2015;16:115-9.

9. Rodrigues A, Sousa-Uva M, Fonseca R, Marques S, Pina N, MatiasDias C. Depression and unemployment incidence rate evolution in Portugal, 1995-2013: General Practitioner Sentinel Network data. Rev Saude Publica. 2017;51:98.

10. Instituto Nacional de Estatística. Dados estatísticos: principais indicadores. Lisboa; 2019. [consultado 10 dez 2019]. Disponível em: https://www.ine.pt/xportal/xmain?xpid=INE\&xpgid=ine_indicadores\&co ntecto=pi\&indOcorrCod=0005599\&selTab=tab0 .

11. Martin-Carrasco M, Evans-Lacko S, Dom G, Christodoulou N, Samochowiec J, González-Fraile E, et al. EPA guidance on mental health and economic crises in Europe. Eur Arch Psychiatry Clin Neurosci. 2016;266:89-124.

12. van de Velde S, Bracke P, Levecque K. Gender differences in depression in 23 European countries. Cross-national variation in the gender gap in depression. Soc Sci Med. 2010;71:305-13.

13. Cortès-Franch I, Puig-Barrachina V, Vargas-Leguás $H$, Arcas $M$, Artazcoz L. Is being employed always better for mental wellbeing than being unemployed? Exploring the role of gender and welfare state regimes during the economic crisis. Int $\mathrm{J}$ Environ Res Public Health. 2019;16:4799.

14. Oyesanya M, Lopez-Morinigo J, Dutta R. Systematic review of suicide in economic recession. World J Psychiatry. 2015;5:243.

15. Drydakis N. The effect of unemployment on self-reported health and mental health in Greece from 2008 to 2013: a longitudinal study before and during the financial crisis. Soc Sci Med. 2015;128:43-51. 